\title{
Ethical appraisal for multi-centre medical research
}

\author{
Sir Michael Drury University of Birmingham
}

Since the end of World War II there has been a requirement for doctors engaged in biomedical research to have their protocols scrutinised by independent ethics committees. The Declaration of Helsinki of 1964, revised in 1975 and again in 1983, set out the broad guidelines and a definitive report on the contribution of the work of ethics committees was drawn up by the Royal College of Physicians of London in 1984. Since then most, but not all, district health authorities in England have established local ethics committees whose role is to scrutinise research on human subjects carried out within their geographical area. Some of these committees, notably those with a teaching hospital within their district, have been busy and have acquired considerable experience in their work. These committees often have members who themselves are very familiar with the problems of research and who also know well the local factors that require consideration. Other of these committees do not have these skills in such measure and may have some medical members with no experience of research method at all. This must handicap them in some ethical issues such as considering the scientific validity of the research to be carried out. With this reservation however, all these local ethical committees are reasonably well placed to scrutinise work within the hospitals in their locality. They are sometimes less well placed to scrutinise research in general practices as some do not have, as recommended, a general practitioner member and sometimes practices have patients in an area served by several district hospitals.

There has been for some years an increase in the biomedical research carried out on patients in the community, some of whom may not be involved with the hospital at all save for, say, the use of supporting laboratory or radiology services. This increase has occurred for several reasons. Firstly, more general practitioners are interested in conducting research. Secondly, more medical problems are dealt with completely or mainly within the community setting

\section{Key words}

Multi-centre medical research; research ethics committees; national ethics committees. and lastly more research of an epidemiological nature is being conducted by community physicians. Most of these studies have the common feature of requiring a wide geographical area to draw patients from as the concentration of the particular problem is less within a community population than within a selected hospital population. This may involve several districts, or a whole region or even become a national study. There are often in general practice studies a large number of practices involved and each one when recruiting patients will need to be satisfied that the protocol has been examined by an ethics committee. For the community physician and the general practitioner this presents the problem of whether ethical approval from one district is a sufficient cover for all patients or whether every district ethical committee has to be satisfied. What happens if one disapproves when the others have approved?

The great strength of the district committee is that it is local. Members may know the standing of the researcher, the circumstances within which he works and local activity that might have a bearing on the research.

There has recently been a proposal that a national ethics committee should be formed to meet some of the problems encountered in multi-centre research in dealing with numerous local committees. There are at least three problems with this. Firstly, it might weaken the authority of local committees. These are seen by most people to be the key feature of our research ethical network and if an all powerful and 'omniscient' committee were to approve then it might be difficult for a local committee to disapprove or, even to have the chance of voicing an opinion. Secondly, many of the studies of a major regional or national size have very special scientific features such as dealing with genetics, or a cultural problem and so on. Until now this has been met by setting up an ad hoc committee with people experienced in the field covered to deal with the particular features. For example, the Royal College of Obstetricians and Gynaecologists has done so for research in the fetal and perinatal field and the Royal College of General Practitioners has a Clinical Research Ethics Committee to consider multi-district drug trials in general practice. This system of ad hoc committees works well and there is no reason why the 
Faculty of Community Medicine should not, if it wished to, establish an independent committee to which epidemiological research over a wide area might be referred. Thirdly there must be a danger of a national ethics committee being drawn into making $e x$ cathedra statements about the wide range of ethical problems, which are often of a very technical nature. There is a difference in concept between an 'ethics committee' which considers the ethical arguments surrounding a major problem and identifies the issues that ought to be considered and that of a 'research ethics committee' which is called upon to make a decision about those issues in a specific case. This distinction could very easily become blurred.

The Conference of Medical Royal Colleges took the view in 1986 and repeated it in 1987 that the disadvantages of a national research ethical committee were such that it should be opposed. Personally I concur with this opinion.

Professor Sir Michael Drury OBE, FRCP, FRCGP, के FRACGP is Head of the Department of General $\vec{\circ}$ Practice, the Medical School, University of Birmingham, Edgbaston, Birmingham B15 2T\}. 